\title{
LES ENJEUX SOCIO-ÉCONOMIQUES DES TECHNOLOGIES DE L'AMÉNAGEMENT DU LITTORAL EN FRANCE \\ CAS DES OUVRAGES DE PROTECTION CONTRE L'ÉROSION MARINE
}

\author{
C. BOUNI (1), P. CHATELLET (1), Y. LAURANS (1) et D. SAUZADE (2) \\ (1) Organisation et Environnement - (2) IFREMER, Toulon-La Seyne
}

\begin{abstract}
The socio-economic implications of the coastal protection technologies in France : case of the protection works against marine erosion.
\end{abstract}

This work is in a part of keeping with the exploratory studies led by IFREMER in view to define their strategy regarding future technological developments in the field of coastal protection. It relies on IFREMER's own reflections and on a study awarded to the company" Organisation et Environnement - Paris " in 1992.

From an overview of the legal context and its trends, the involved actors were identified and the socio-economic implications of this sector were assessed. These analyses were supported by the results of an inquiry among the institutions, public utilities, laboratories and design offices to coastal protection in France.

The laws which rules this field are numerous and fit to cope with the pressure that is exerted in many ways on the coastal area. Yet, the importance of this legal instrument results in texis being sometimes imprecise or even contradictory. The law of 1986 and its applicative order of 1989 have progressively led to enforce a global management policy, taking into account the preservation of the coastal ecosystem, which was ratified by the
" Coastal Law" of 3 January 1992.

Within this evolutionary context, the decision making. and financing lines of the coastal protection sector have been identified. They articulate around the key institution which are the Maritime Services of the District.

The French State support has been severely cut down thus leaving to the territorial organizations to manage and finance the coastal protection. The territorial organization spending for this purpose reaches 100 million French Francs over one year for France itself.

The private bid as far as coastal studies are concerned was traditionally assumed by well-known laboratories such as SOGREAH, LNH (Laboratoire National d'Hydraulique), BCEOM... They are now frequently in competition with local engineering companies although the total annual spending for the coastal studies does not exceed 8 to 10 million $F F$.

The techniques in use remain conventional and the works are often realized to solve local and specific problems without considering the possible impacts on the adjacent sectors. Most of the works are rock-built.

If the present trends are confirmed, the coastal areas should grow more fragile, although being considered as a priority, without leading to a significant improvenent of the techniques in use.

Cette recherche s'inscrit dans une démarche exploratoire de l'IFREMER dans le domaine des technologies de protection du littoral. Elle a pris appui sur une étude confiée en 1992 à la société Organisation et Environnement qui a réalisé une enquête auprès des institutions, services publics, laboratoires et bureaux d'études concernés par la protection de côtes contre l'érosion marine.

A partir d'une analyse du contexte juridique, la méthodologie suivie a consisté à identifier les acteurs et à évaluer les enjeux socio-économiques de ce secteur ainsi que ses perpectives d'évolution. 
Cette recherche s'inscrit dans une démarche exploratoire de l'IFREMER dans le domaine des technologies de protection du littoral. Elle a pris appui sur une étude confiée en 1992 à la société Organisation et Environnement qui a réalisé une enquête auprès des institutions, services publics, laboratoires et bureaux d'études concernés par la protection de côtes contre l'érosion marine.

A partir d'une analyse du contexte juridique, la méthodologie suivie a consisté à identifier les acteurs et à évaluer les enjeux socio-économiques de ce secteur ainsi que ses perpectives d'évolution.

\section{INSTRUMENTS JURIDIQUES DE LA PROTECTION DU LITTORAL}

\section{A- CADRE INTERNATIONAL}

On constate que le milieu marin et les espaces littoraux font l'objet d'une attention particulière depuis les années 1960/1970 du point de vue des normes internationales. Les textes à caractère général ont été le plus souvent complétés ou renforcés par des accords régionaux et/ou thématiques dans les années 1980.

En effet on observe, du point de vue des normes juridiques, une transition :

A partir de textes très généraux fondateurs, qui posent des pétitions de principes de protections, on est arrivé à des textes spécifiques à une aire géographique ou à des thèmes ciblés visant à rendre la vrotection des milieux effective. Cette transition s'est produite sur une période de 20 ans (1960 à 1980). Elle devrait donner lieu dans les décennies à venir à la poursuite de cette identification des milieux. Celle-ci pourrait être complétée par des objectifs concrets de gestion globale des milieux, de protection du littoral et de lutte contre la pollution.

A cet égard, le cas de la Camargue est significatif puisqu'elle cumule les protections par l'inscription aux réserves du MAB (protection régionale) et à la convention RAMSAR sur les zones humides (protection thématique) tout en bénéficiant au titre national du statut de parc naturel régional et, pour partie, de réserve naturelle (protections régionales et thématiques).
Lapproche internationale du theme de la protection ol licapproche internationale di theme de la protection du littoral permet de degager un cetain nombre de textes fondamentaux visant aiss bich la protection el la conservation du milieu et des ressources que $1 \mathrm{a}$ tute contre la pollution Cet arsenal juridique se decompose dune part en Convent ons a objet general

La convention de Geneve du 29 avil 1958

Ia convention des Nations Unies sur le droit de la net conclue 2 Montego Bay le 10 décembre 1982

Laccord du $2111-83$ pour la mise en place d'un teseau etropeen expenmental de stations oceaniques.

Conventions a vocation pariculiere et ou regionale

Reserves du MAB (Man and Biosphere) de IUNESCO Son 1 objet est de protéget sinultanennent le milieu naturel et son environnenent humain, parta techerche dune complémentante entre environnenent et developpenent

Convention de Ramsar, 1971 Elle vise a la protection des zones humudes d'importance internationale, pour lesquelles les Etats signatares sengagent a elaborer et nelfre en oeuvre des plans d'amenagement destines a favoniser leur conservation

Aires proteg ees au fitre des protocoles et convertions des mers regionales du PNUE

Aver, en ce quil concerne la France,

Mediternanee

Convention du $18-4-1951$ sur lorganisatjon europeenne et méditerrane enne pour la protection des plantes.

Convention cadre de Barcelone du $16-021976$

$\mathrm{Accords}$ franco-italo-monegasques sur la protection des eatu $\mathrm{du}$ littoral du 10 mal 1976

Protocole difthenes du $1705-1980$ relatif a 10 protection ce la mediterranee contre les pollutions dongine tellurique.

Protocole de Geneve du $3-041982$, relatif aux atres spectalennent protégeess

Caraibe

Convention de Cartagena de Indias du $2403-1983$ pour la protection t ta mise en valeur du milieu marin de la région des caratbes

Oceanindien.

Convention el protocole de Nairobi du $17 \& 21 \% 06-1985$ pour la protection, la gestion et la mise en valeur du milieu marn et des zones cotieres de la régon de lafrique orientale,

et des zones protégées ains qu'a la faune et à la flore sauvage.

Pacifique sud:

Convention de Nouméa du 24-11-1986 pour la protection des ressources naturelles et de lenvironnement.

Aflantique:

Convention intemationale de Paris du 4-06-1974 relative 2 la prevention de la pollution marine d'origine tellungque conclue entre les pays riverains de l'atlantique nord-est.

Convention et protocole d'Oslo des 15-02-1972, et 2-03-1983 pour la prevention de la pollution marine tésuhant des operations d'immersion 


\section{B - CADRE NATIONAL}

- La protection du littoral liée au droit de l'urbanisme

La loi "Littoral" du 03-01-1986 introduit plusieurs articles 1 codifiés sous le chapitre VI du Code de l'urbanisme. Ce chapitre a valeur de "loi d'aménagement et d'urbanisme", et aussi de "prescription nationale applicable à certaines parties du territoire". Ses dispositions s'imposent donc à l'ensemble des documents d'urbanisme qui doivent être compatibles ou mis en compatibilité avec elles. Une instruction du 24-10-1991 a été prise pour expliciter les dispositions de la loi-littoral et va plus loin que le texte, en insistant notamment sur deux points :

- le rôle de l'Etat garant du respect des obligations fixées par la loi,

- la nécessité d'une approche intercommunale des questions de protections du littoral.

L'approche intercommunale conduit à évoquer les instruments de planification spécifiques au littoral : des démarches de planification supracommunale avaient été lancées dès les années 1970 (les schémas d'aménagement du littoral). Toutefois, jusqu'aux schémas de mise en valeur de la mer (SMVM) et aux schémas d'aménagement régionaux (SAR), aucun de ces documents n'avait de valeur juridique.

Evoquons, enfin, une disposition "antimitage" largement sous-exploitée par les collectivités locales: certains articles du Code de l'urbanisme donnent d'importants pouvoirs au maire ou au préfet pour s'opposer aux divisions foncières susceptibles de "compromettre gravement le caractère naturel des espaces, la qualité des paysages ou le maintien des équilibres biologiques auxquels participent ces espaces". (L. 111-5-2 et R. 315-55 et suivantes du Code de l'urbanisme).
Linstruction du 24-10-1991 explicite certaines des dispositions de to $10 \%$ inserées au code de lurbanistre, mais également certaines artscles de la loi littoral qui ne sont pas inseres dans le Code Elle a ete mise en oeuvre pour partie par la circulaire di 28-051992.

Eile aborde la question toujours sersible de la fjustification économique des projels d'aménagetnent littoraux Cette approchecest novatrice car, jusqualors, aucun texte de doctrine admunistrative ravait aborde le probleme en matiere de protection du littoral.

Linstruction aborde egalenent les questions plus techniques liees a a définition do coupure durbanisation $(1 / 46-2)$, d extension de lutbanisationt et création de nouveaux hameaux $*(141464)$, de rapacte d accueil et despaces a preserver $(-146-6) \mathrm{Ce}$ dernier article a fait lobjet de la parution d'un dectet en date du 20 septerb bre 1989 et de deux circulaires d application des 10 et $25.10-1989$.

Pour autant un certain nombre d ambigutes ef de difficultes ne sont pas levees, 1 o creculaire de 1992 nevo quant pas ces questions.

Cest sur ces notions que les discussions urisprudentielles actuelles sont les plus rives, faute de criteres precis d appreciation od ou de nombreux jugements parfois contradictoires sur le sujet):

Enfin Iinstruction rappelle les principes régissant lutilisation du domane public maritime

1tes Schemas de Mise en Valeur de la Mer' definusent les onentations fondamentales de la protection de lexploitation el de lamenagenent dulittoral

Les SMV VM dolvent porter sur des zones cótieres, dans toutes leurs interactions et d ynamiquest, ce qui est plus imprecis que lo champ d'application de $1 \mathrm{a}$ loilittoral 11 convient cependant que le pernometre du SMVM constitue une unite geographique et maritume et presente des interets lies, concurrents ou complémentaires ${ }^{2}$ Une narge dappreciation inportante existe done pour son conteril et pour le prefet qui fixele perimetre du schema. Linteret porté en genteral au SMVM depend dono directement de limportance gue leur donne $1 e$ prefet lors de son elaboration

Enfin, les departements d'outre-mer sont pour leur part pourvus dinstruments specifiques 1 es $S A R^{2}$

1/Les SMVM ont ete consactes par laricle 57 de la lo 7 lanvier 1983, modifiee par liaricle 18 de la loi littoral, et le decret du 5 decembre 1986

${ }^{2}$ Leur regime juridique est fixe par la loi du 2 aoù 1984, et le decret du 20 août 1988 
- La protection du littoral en dehors du droit de l'urbanisme

De nombreuses dispositions juridiques, non directement liées au droit de l'urbanisme, étoffent considérablement l'arsenal juridique relatif à la protection du littoral. Ces dispositions visent la protection du domaine public maritime.

Le domaine public maritime est une composante essentielle du littoral. Il est soumis à un régime protecteur: principes d'inaliénabilité, d'imprescriptibilité, de liberté et de gratuité d'accès.

On signalera que 3 circulaires généralement peu connues et qui n'ont pas été abrogées peuvent s'avérer précieuses lorsque des travaux ou aménagements ne sont pas soumis à la procédures d'étude ou de notice d'impact ou à une procédure d'enquête publique.

En dehors de ces textes, on doit mentionner à nouveau la loi-littoral ${ }^{l}$ qui en dehors de ses volets "urbanistes" veille à la protection du littoral notamment en matière de décision d'utilisation du domaine public d'autorisation d'occupation et de réglementation d'activités, parmi lesquelles les activités extractives et les activités de cultures marines.

Il convient, par ailleurs, de souligner les principes fondamentaux suivants consacrés par la loi :

- l'accès du public au rivage

- l'accès du public au cheminement le long du littoral.

- l'accès du public aux plages

A ces textes, s'ajoutent et s'entremêlent de façon délicate d'autres textes que nous ne détaillerons pas ici en raison de leur thème très spécifique portant sur la circulation des véhicules terrestres et les épreuves ou compétitions sportives.

3 circulatres insuffisamment exploiteces

Ta, circilare du 10 novembre 1972 relative a retude biologique systematique a locasion des projets damenagenent dulittoral,

t la circularie du 26 novernbre 1973 relative a la consultation des experts en écologie a lechielon regiond pour Ia menagenent du littoral, des principaux lacs naturels et des grandes retenues artificielles,

-1. la circulaire du 26 novernbre 1973 relative a la menagenent dulittoral mantine et des lacs importants:

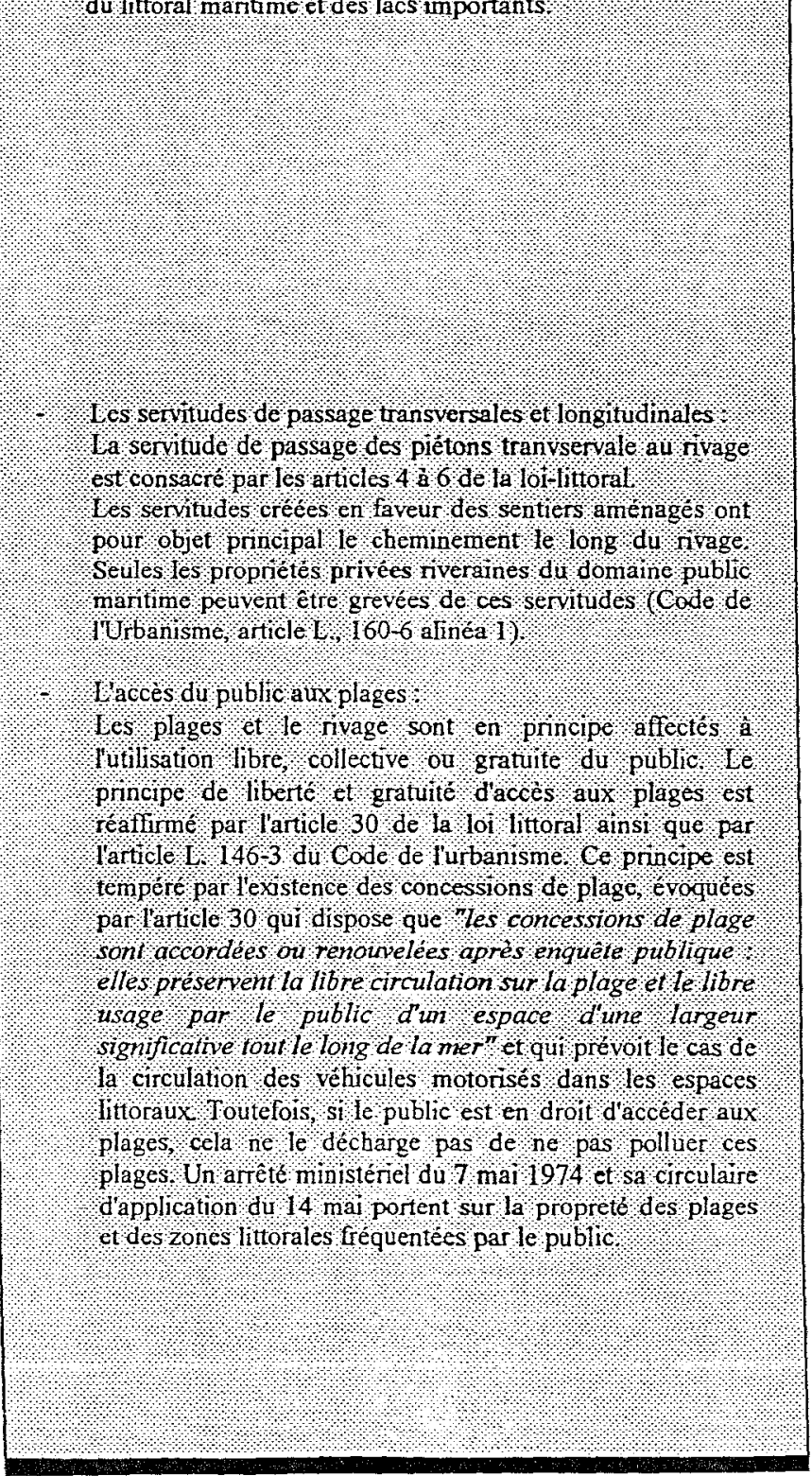


Enfin, nous ne saurions ignorer dans le cadre de cet exposé la mention de l'action de protection du lițtoral exercée par le Conservatoire par le biais de ses acquisitions foncières. L'Office National des Forêts lui apporte son concours technique, pour l'entretien et l'aménagement des espaces boisés.

- L'illustration concrète de la mise en oeuvre d'un arsenal juridique de protection sur les thèmes des rejets en mer de l'érosion marine :

\section{$\rightarrow$ Rejets en mer :}

Ils doivent faire l'objet d'autorisation préalable, dès lors que ce ne sont pas des rejets provenant d'installations classées. Tous les rejets (déversements effectués dans les eaux de mer territoriales ou sur le domaine public maritime ou, plus généralement, tous faits susceptibles d'altérer la qualité des eaux de mer sont soumis à autorisation.

$\rightarrow$ L'érosion marine :

Depuis La loi du 16 septembre 1807, ce thème fait l'objet d'une attention particulière. Pour sa part, la loi sur l'eau du 3 janvier 1992 habilite les communes à engager les études, l'exécution et l'exploitation de tous travaux, ouvrages ou installations présentant un caractère d'intérêt général ou d'urgence, dont les travaux de défense contre les inondations et contre la mer (article 31 de la loi du 3 janvier 1992, décret du 21 octobre 1993). Cette loi constitue un outil capital en vue de la gestion globale de la ressource "eau". Elle reprend à son compte les dispositions éparses touchant à ce domaine, dont certaines relatives au littoral. Le littoral fait ainsi désormais, à part entière l'objet d'une préoccupation qui intégre l'interaction entre les divers milieux pouvant influencer sur sa préservation et sa mise en valeur, ceci tout en veillant au respect des intérêts des différents acteurs qui l'occupent.

En définitive, on peut se demander si, plutôt que de prétendre lutter contre les effets de la mer, il ne convient pas de composer avec elle. S'il paraît difficile de ne pas tenir compte de l'héritage du passé dans ce domaine, cette question doit se poser pour tous les aménagement à venir. Il incombe donc aux communes, acteurs en première ligne, d'assurer préventivement la maîtrise des activités terrestres (notamment dans leurs documents d'urbanisme) afin d'être "en avance d'une loi "pour préserver leur environnement et leur développement futur par les moyens juridiques dont elles disposent à ce jour.
La gestion et lamenagenent du site de la pointe des chateaux en entre Conservatoire du littoral et loffice National des Forets.

Les differents types de rejets

les rejets industriels, soumis soit a la réflementation applicable aux rejets des installations classes, soit a la reglementation generale du decret $\mathrm{n}^{\circ} 73-218$ du 23 fevrier 1973 .

- les rejet domiestiques, sont en principe soumis a autorisation. IIs en sont cependant souvent exenptés en application de larrete du 13 por 1975 (article 5), les seuils fixes par cet arreté nétant generalement pas depasses par ce genre de rejet.

- les rejets agricoles, au fitre de la police des eaux de ner, 1 rexiste pas de regles specifiques aux rejets des installations agricoles sl ces rejets proviennent d'installations classees Silel reest pas le cas, ils sont soumis aux dispositions du decret $\mathrm{n}$ ? 73218 du 23 ferrier 1973

4 les rejets des agglomerations littorales 1 s sont soumis autonsation comine tout autre déversement. Les conditions techniques sont definies par larreté du 20 novembre 1979,14 circulaire du 101 in 1976 et linstruction du 12 mal 1981

L Les rejets des zones durbanisation future des communes littorales. lurbanisation ne peut se faire que soins eserve de Texistence de traitement et d'evacuation des effluents conformes ala réglenentation issues de la police des eaux ou de dispositifs autonomes (ot littoral, articlel7)

Tartide 33 dela loi du 16.091807

Larticle 33 toujours en vigueur, dispose que torsquil sagira de constrime des digues a la mer, ou contre les fewes. ntieres el tortents navigables ou non navigables, la nécessité en sera constalee par le gouvernement el 10 depense supportee par les propnétés protégées, dans la proportion de leur interet aur travau, sauf le cas ou le gouremenent croirait utile et fuste daccorder des secours sur les fonds publicst

Travaux de defense et trude d impact:

Le décret du 5 revrier 1971 , modifié par le decret du 12 octobre 197 , concene lautonsation de defense des lieux habités contre la mer. Ainsi, les travaux de défense sont pris en consideration et autorisés par une décision du prefet $\mathrm{L}$ dossier de travaux comprend une etude dimpact lorsque le cout total des ouvrages a realiser excede $12 \mathrm{MF}$ ou lorsque lemprise depasse 2000 metres carres Guadeloupe coistitue un exemple de cooperation exemplaire 


\section{LE SECTEUR DE LA PROTECTION DES CÔTES.}

\section{A- LA SITUATION PRÉSENTE : 7 POINTS CAPITAUX}

\section{Un désengagement de l'Etat}

La "demande" des collectivités territoriales en matière de protection du littoral est tout d'abord marquée par un contexte de désengagement de l'Etat, dont les subventions se sont considérablement réduites ces dernières années, jusqu'à tomber probablement à 0 cette année, sauf tempête exceptionnelle, et sans préjuger d'une utilisation éventuelle de la réserve parlementaire, qui en 1992 a permis d'attribuer cinq millions de francs à ce titre de la Loi de Finances.

\section{Une redéfinition des rôles}

Ce désengagement de l'Etat s'explique d'une part par l'austérité budgétaire actuelle, mais il est aussi lié à la redéfinition des attributions des collectivités territoriales, qui voient leur responsabilité accrue dans ce domaine. Les orientations futures, en ce qui concerne le partage des tâches entre Etat et collectivités ont fait l'objet d'une importante réflexion sous l'égide du Conseil Général des Ponts-et-Chaussées. Ces recommandations, et notamment celles qui proposaient que l'Etat prenne à sa charge les études scientifiques de base, contribue à l'élaboration de plan régionaux de programmation sous forme de "Schémas techniques globaux", et incite à la réalisation d'opérations exemplaires, ont peu été suivies jusqu'à présent, faute de moyens suffisants mis à la disposition des ministères concernés.

\section{Une pratique actuelle parcellisée}

La pratique courante de la protection du littoral s'organise essentiellement à l'échelle départementale, avec les Directions Départementales de l'Equipement et les Services Maritimes. Cette protection se fait par une succession d'opérations ponctuelles, d'un linéaire rarement supérieur à 100 mètres, et sans qu'il soit fait un usage fréquent d'études océanographiques circonstaneiées.

\section{Financement : de la règle des quatre quarts à la règle des trois tiers}

Jusqu'alors le financement des opérations se partageait approximativement entre quatre bailleurs : les collectivités territoriales locales, la Région, les propriétaires riverains, l'Etat. Ces dernières années, les trois premiers types de bailleurs de fonds seulement participaient significativement à ces efforts.

5. Une dépense annuelle de $\mathbf{1 0 0}$ millions de francs

La dépense annuelle pour la protection du littoral contre l'érosion marine en France représente un montant relativement stable de 100 à 120 millions de francs, bien que sa répartition soit assez variable. La Méditerranée représente 10 à $15 \%$ du total, la Manche $35 \%$, l'Atlantique 45 à $55 \%$ du total. De cette dépense totale on peut déduire que la demande d'études devrait être en tout état de cause inférieure à 10 millions de francs par an.

6. Etudes techniques : une offre concentrée, une activité marginale

L'offre, en termes d'études littorales, est représentée au plan national par quelques organismes importants comme SOGREAH, le BCEOM, le LNH... pour lesquels le secteur de la protection des côtes n'est qu'une activité marginale. Ainsi le chiffre d'affaires du département "Ports et Côtes" de SOGREAH serait à lui seul aussi important que le volume sconomique que représente le secteur en France ; cela conduit à penser que ce chiffre d'affaires est vraisemblablement réalisé essentiellement sur le secteur des ports. Ces organismes sont de plus en plus mis en concurrence avec des bureaux d'étude locaux ou des structures universitaires. Pour des opérations importantes, les régles Européennes ont 
élargi l'offre aux grands laboratoires européel comme Delft Hydraulics, Wallingford, le Danish Hydraulic Institute...

\section{Un besoin de protection du littoral potentellement important}

L'analyse de la situation du littoral vis-à-vis de l'érosion fait apparaître un besoin a priori important en protection ( $37 \%$ du littoral recule de plus de 0,5 mètre par an). Les équipements installés couvrent une faible partie du littoral (10\% environ), et sont réalisés en enrochements $(32 \%)$ ou en maçonnerie (27 $\%$ ).

\section{B - LES PRÉVISIONS DE TENDANCE : UNE RELATIVE STABILITÉ...TANT QUE LE TEMPS RESTERA CALME}

1. Une approche plus globale des problèmes?

La prise de conscience de la nécessité d'aborder la protection du littoral de façon plus globale que par le passé devrait susciter la réalisation d'études aboutissant à la définition d'une stratégie d'aménagement portant sur un linéaire de littoral suffisamment important pour comprendre les phénomènes en jeux. $\mathrm{Ce}$ type d'étude est en cours de réalisation sur les littoraux du Cotentin, de la Manche et de Camargue. Elles sont généralement financées en association par les collectivités territoriales, l'Etat et dans certains cas la Commission Européenne.

\section{Un littoral qui se fragilise}

Il semble que l'avenir devrait voir le littoral de plus en plus fragilisé par l'érosion marine du fait de l'action de plusieurs facteurs qui se conjuguent (réduction des apports sédimentaires, élévation du niveau de la mer, impact des aménagements existants...), alors que les pressions économiques sur cette zone augmenteront. Cependant, une volonté politique de prise en compte de la question de la protection du littoral est manifeste et devrait se répercuter dans les textes. Le littoral devrait apparaître à l'avenir comme un espace prioritaire.

3. Une prédominance des enrochements, dans la pratique passée et à moyen terme

Il ne semble pas qu'une évolution notable soit à signaler du point de vue des techniques de protection des côtes : les ouvrages en enrochements restent majoritairement employés, et l'utilisation de procédés nouveaux, pour l'instant marginale, n'a que peu de chances de s'imposer massivement, du fait d'une part des contraintes dues au milieu physique, et d'autre part de la faible adhésion a priori que remportent ces procédés auprès des responsables de l'ingénierie des ouvrages.

4. Une opinion publique qui réagit surtout après le passage des tempêtes

Les pressions liées à l'opinion publique pour une augmentation de la protection des côtes sont associées au passage des tempetes, et ne devraient pas stimuler de manière significative une modification des pratiques actuelles.

\section{C- CONCLUSIONS :}

L'analyse juridique fait apparaître une perspective de renforcement de la protection du littoral du point de vue de l'environnement et de l'urbanisme. Ce renforcement entraîne une plus grande exigence vis à vis de l'insertion dans l'environnement des aménagements. En conséquence il devrait y avoir sur le littoral moins de projets, tendance encore renforcée par la conjoncture économique actuelle, mais de plus grande qửalité.

Malgré une fragilité croissante du littoral, le volume total des études consacrées à la protection du 
littoral reste faitere, de l'ordre de 10 million de francs, et ne devrait pas évoluer dans l'immédiat, sauf catastrophe.

Cependant, la demande en matière d'études scientifiques de base et d'élaboration de schémas d'aménagement à l'échelle régionale devrait croitre, sous l'impulsion des Régions, de l'Etat et éventuellement de la Commission européenne.

Le contexte général n'est pas très favorable au développement de nouveaux procédés, qui ne font pas en France l'objet d'efforts soutenus de recherche.

Toutefois, la progression des connaissances en hydrodynamique et en sédimentologie côtière, l'exemple de pays étrangers comme le Japon ou les Etats Unis laissent imaginer une introduction progressive de procédés nouveaux ou de variantes innovantes de procédés conventionnels.

Ces nouvelles techniques trouverons à s'appliquer dans la mesure où elles pourront offrir des garanties en matière d'efficacité et de pérennité. L'exiguité du marché national exige d'amortir les coûts de développement à l'export.

Les applications faisant appel aux techniques nouvelles vont d'abord concerner les projets d'aménagement importants destinés au tourisme, où l'aspect insertion dans l'environnement est primordial.

\section{Références :}

- Etude des enjeux socio-économiques concernant les technologies de l'aménagement du littoral, Organisation et Environnement (C. Bouni, P. Chatellet, Y. Laurans) pour IFREMER, 120 pages hors annexes, Déc. 1992.

- Rapport concernant les travaux de lutte contre l'érosion marine, Conseil Général des Ponts et Chaussées, Groupe de travail présidé par M. P. Aridrau, Janv. 1990.

- Etude de la problématique des dispositifs de protection du littoral à impacts réduits sur l'environnement, SOGREAH (B. Bellesort) pour IFREMER, 100 pages hors annexes, Mai 1993.

- L'aménagement du littoral, document DATAR, La Documentation Française, 1993. 